\section{Re: Electronic Health Record Challenges, Workarounds, and Solutions Observed in Practices Integrating Behavioral Health and Primary Care}

To the Editor: Cifuentes et $\mathrm{al}^{1}$ address the important issue of primary care behavioral health documentation and communication in electronic medical records. The article by Cifuentes et al reports frequent use of workarounds to respond to the inability of practices to integrate their electronic health records (EHRs). They note that at the end of the study, 2 of 11 practices were developing unified EHRs.

Though based on a limited convenience sample of practices, their conclusion leads readers to assume that in integrated care, EHR issues are not fully resolved. There are 3 concerns with the conclusions: EHR issues are not fully developed in many areas of medicine; integrated care cannot function without integrated EHRs; and multiple examples of well-developed, transparent, bidirectional EHRs exist in multiple integrated family medicine practices around the country.

EHRs are still in their youth (or adolescence), and have not yet grown into the hopes that many have for them. Templates for documentation, ease of use, interoperability, ability to extract data fields, use as part of care algorithms, and responding to the multiple needs for functionality for a broad range of users are among the current issues being considered. Applications for behavioral care within EHRs suffer the same and perhaps greater frustrations of all EHR users: how to use the available technology to meet their needs. These frustrations are often administrative rather than technical issues. The priority and pressure for developing integrated behavioral care systems is just emerging.

The degree of process integration among practices with co-located or integrated care varies widely. Summary data from the Practice Improvement Profile (PIP), a measure of the degree of practice integration derived from the Peek Lexicon, note that total scores on integration implementation show a median of 57.7 (range, $0-100$ ), and that communication between behav- ioral health and primary care has a median score of 75 (range, $0-100$ ). As we try to identify the core elements of integrated behavioral care, there are those who suggest that co-locating a clinician in a primary care practice is necessary, but is not sufficient to be considered integrated care. ${ }^{2,3}$

There are multiple examples of practices and systems that include transparent, retrievable, templated, bidirectional behavioral communication in their EHRs. Work at Oregon Health Sciences University, Cherokee Health Systems (Tennessee), and Southeast Community Health Systems (Alaska) suggest that some early adapters have overcome most of the obstacles reported. In the recently funded Patient-Centered Outcomes Research Institute's Integrated Behavioral Health in Primary Care trial, the 40 participating practices must be developing integrated EHRs as a condition of participation.

In summary, a behavioral presence in EHRs is associated with the same growing pains as any other EHR user groups, amplified by the recency of integration development. The issue of whether colocated practices without EHR access, or integrated care with integrated EHRs, generate different outcomes is an important question that we hope to respond to in our trial, but until then, like most new initiatives, there is a range of responses to the challenges. Cifuentes et $\mathrm{al}^{1}$ report a group of practices struggling with EHR integration, whereas many other practices have resolved these challenges and have functioning bidirectional EHRs.

Rodger Kessler, PhD

Department of Family Medicine University of Vermont College of Medicine, Burlington

Behavioral Health Program Arizona State University

National Research Network American Academy of Family Physicians Juvena R. Hitt, BS

Department of Family Medicine University of Vermont College of Medicine, Burlington

\section{References}

1. Cifuentes M, Davis M, Fernald D, Gunn R, Dickinson P, Cohen DJ. Electronic health record challenges, workarounds, 
and solutions observed in practices integrating behavioral health and primary care. J Am Board Fam Med 2015;28(Suppl 1):S6372.

2. Kathol RG, Degruy F, Rollman BL. Value-based financially sustainable behavioral health components in patientcentered medical homes. Ann Fam Med 2014;12: $172-5$.

3. Peek CJ; National Integration Advisory Council. Lexicon for behavioral health and primary care integration: concepts and definitions developed by expert consensus. AHRQ publication no. AHRQ 13-IP001-EF. Rockville, MD: Agency for Healthcare Research and Quality; 2013. Available from: http:// integrationacademy.ahrq.gov/sites/default/files/Lexicon.pdf. Accessed January 15, 2016.

doi: 10.3122/jabfm.2016.02.150355

The authors of the original article are in agreement with the authors and declined to comment.

\section{Re: Patient Preferences for Receiving Reports of Test Results}

To the Editor: The recent report by LaRocque et $\mathrm{al}^{1}$ titled "Patient Preferences for Receiving Reports of Test Results" is very interesting. The authors mentioned that "participants demonstrated preferences in how they received test results by non-in-person communication methods, preferring personal E-mail and password-protected websites, but they did not prefer fax. ${ }^{1}$ "Of interest, the new information technology seems to be a useful technology for laboratory result distribution. However, there are many concerns about the system. First, in laboratory medicine, quality control of the postanalytic phase has to cover result reporting. ${ }^{2}$ There must be the system to validate the correctness of the results in the IT system. Second, privacy and data protection become important issues. We must be well prepared to prevent hackers from hacking the database and laboratory resultreporting IT system. ${ }^{3}$ Third, it should also be noted that some patients might not want to know their results, and this is their right. ${ }^{4}$ There must be a specific operation for not uploading the data for these cases into the IT system.

Viroj Wiwanitkit, MD, FRFM

Surin Rajabhat University Surin, Thailand wviroj@yahoo.com

\section{References}

1. LaRocque JR, Davis CL, Tan TP, D'Amico FJ, Merenstein DJ. Patient preferences for receiving reports of test results. J Am Board Fam Med 2015;28:759-66.

2. Walz SE, Darcy TP. Patient safety \& post-analytical error. Clin Lab Med 2013;33:183-94.

3. Tisserand IN. [New risks of addiction for new populations: the example of hackers]. Ann Med Interne (Paris) 2000; 151(Suppl B):B49-52.

4. Wiwanitkit $\mathrm{V}$. Right not to know the investigation result. J Med Assoc Thai 2008;91:595-6.

doi: 10.3122/jabfm.2016.02.150367

The authors of the original article are in agreement with the author and declined to comment. 